\title{
Adaptable and Adaptive Information Provision for All Users, Including Disabled and Elderly People
}

\author{
Josef Fink, Alfred Kobsa \\ GMD FIT - German National Research Center for Information Technology \\ Human-Computer Interaction Research Division \\ D-53754 Sankt Augustin, Germany \\ Phone: +49 $224114\{2729,2315\}$ \\ Fax: +49 $224114\{2065,2167\}$ \\ E-mail: \{josef.fink, alfred.kobsa\}@gmd.de \\ Andreas Nill \\ USW, Schloss Gracht, D-50374 Erftstadt, Germany \\ Phone: +492235 406219 \\ Fax: +49223517369 \\ E-mail: nill@usw.de
}

\begin{abstract}
Due to the tremendously increasing popularity of the World-Wide Web, hypermedia is going to be the leading online information medium for some years to come and will most likely become the standard gateway for citizens to the "information highway". Already today, visitors of web sites are generally heterogeneous and have different needs, and this is likely to increase in the future. The aim of the AVANTI project is to cater hypermedia information to these individual needs by adapting the content and the presentation of web pages to each individual user. The special needs of elderly and disabled users are also partly considered. A model of the characteristics of user groups, individual users and usage environments, and a domain model are exploited in the adaptation process. One aim of this research is to verify that adaptation and user modeling techniques that were hitherto mostly used for catering interactive software systems to able-bodied users also prove useful for adaptation to users with special needs. Another original aspect is the development of a network-wide user modeling server that can concurrently accommodate the user modeling needs of several applications and several instances of an application within a distributed computing environment.
\end{abstract}

Keywords: Adaptive hypermedia, individualization, personalization, adaptability, adaptivity, disabled users, user modeling, user model server

\footnotetext{
${ }^{1}$ The research described here has been partly funded by the European Commission in the ACTS programme. We would like to thank the three anonymous NRMH reviewers for their valuable recommendations, and Gabi Nordbrock and Rüdiger Hüttenhain for their assistance in preparing this paper.
} 


\section{Introduction}

The aim of the AVANTI project (1) is to develop and evaluate a distributed information system that provides hypermedia information about a metropolitan area (e.g., about public services, transportation, buildings) for a variety of users (e.g., tourists, citizens, travel agency clerks, elderly people, blind persons, wheelchair-bound people, and users with (slight) forms of dystrophy ${ }^{2}$ ) with different interests, knowledge, and abilities. The system can be accessed through the World Wide Web (WWW) from people's homes, travel agencies, public information kiosks and on the go, where different hardware platforms, software environments, network speeds, and environmental surroundings can be anticipated. In order to cater to the different user needs and usage environments, AVANTI exploits methods and tools developed in the context of adaptive and adaptable systems during the last few years. Internal models of user groups, individual users, usage environments, and a domain model help adapting the content and presentation to each user's individual needs.

Until recently, existing adaptable and adaptive systems considered mostly users with "normal" physical, sensorial, and cognitive abilities only (see however (2, 3, 4, 5) for recent exceptions). People with special needs, like the disabled and elderly, should however also be given the opportunity to take advantage of computer-mediated information. Such information is already indispensable at many workplaces, and increasingly also becomes relevant in public places and at home. Access to information in hypermedia form is particularly important since it is likely to become the standard gateway to electronic information and entertainment.

Computer access for people with special needs has therefore been a research issue since many years. Considerable efforts have been put into making software systems usable by people for whom they were originally not designed (e.g., access to graphical user interfaces for visually or manually impaired users) and into developing databases with dedicated information for the disabled (e.g., information on wheelchair accessibility of public transportation, or verbal descriptions of paintings in major museums) which supplements already available data collections that do not take people with special needs into account. These solutions are mostly restricted to very small populations of disabled users and are therefore usually fairly expensive due to the small number of potential customers. It seems, however, that techniques for adapting generic interactive software systems to the needs of individual user groups can be employed to also tailor software systems to the needs of many disabled and elderly people. This approach not only seems theoretically more satisfactory, but may also be economically more viable than isolated dedicated solutions.

\section{User Needs}

Investigations on user needs for hypermedia information systems that were reported in the literature, or carried out in the context of AVANTI through interviews among tourists, citizens and people with several types of disabilities (6), showed a considerable heterogeneity between these groups, to which information systems should cater. Moreover, individual differences in

\footnotetext{
${ }^{2}$ Dystrophy is a range of circa 40 neuromuscular diseases resulting in muscle weakness, paralysis, cramps, impaired muscle relaxation, etc.
} 
user needs have also been encountered. Here are a few examples to illustrate this point (many others will be discussed in the remainder of this paper):

- For blind users, the modality of the presented information must be changed to tactile and/or audio output. Moreover, supplementary orientation and navigation aids, like an additional table of content containing all internal and external links on a hypermedia page, are helpful for this user group (7).

- For wheelchair-bound users, detailed information concerning the accessibility of buildings (e.g., the existence and the dimensions of ramps and elevators, the type and width of doors) is important and should therefore be automatically provided. This information is redundant for able-bodied users and should be omitted for them (6).

- For users interested in a specific subject, more detailed information should be provided, e.g. information about the history of an important sacral building. If the user lacks this specific interest, such information should not be presented in order to lower the efforts necessary for building a mental model of the current hypermedia page $(8,9,10)$.

- For computer novices, usage instructions should be augmented by an explanation. Normally, this is not necessary for experienced computer users.

- For users with low-bandwidth network access (e.g., via a slow modem), information with high data volume (e.g., videos, high-resolution pictures) should be replaced by less demanding, but nevertheless appropriate, equivalents in order to reduce download times. The response time of a hypermedia system is extremely critical from a usability point of view (11).

Since all these needs cannot be addressed within a single project, AVANTI focused mainly on mobility-related user requirements.

\section{Scope of Adaptability and Adaptivity}

In order to cater to different user needs, information systems can be tailored manually by the system designer (and possibly the user) or automatically by the system. Systems that allow the user to change certain system parameters, and thereby adapt the behavior of these systems, are called adaptable. Systems that adapt to users automatically based on monitoring the users' interaction during runtime are called adaptive (12). Both features, adaptability and adaptivity, are provided by the AVANTI system on two different levels:

\section{- Adaptability and adaptivity at the user interface}

This includes special I/O devices (e.g., macro mouse, Braille display, speech synthesizer), visual and non-visual interface objects, and associated interaction techniques $(13,14)$.

- Adaptability and adaptivity within hypermedia pages

This includes the adaptation of the information content, information modality, information prominence, orientation and navigation aids, and links to other hypermedia pages (15).

Whereas the first group of adaptations aims at enabling and improving the overall access to the information system, the second group of adaptations focuses on the personalization of one specific hypermedia system. In the rest of this paper we will focus on the latter goal since this 
seems to be a rather novel approach which complements existing solutions to provide access to the WWW for everyone, including users with disabilities.

\section{Overview of the System Architecture}

In this section, we will give a brief overview of the principal components of AVANTI, and the interaction between them. Those components that are central to the aims of this paper will then be described in more detail in the subsequent sections.

The main components of the AVANTI system are (see Fig. 1):

a) Multimedia Databases with information about the AVANTI domain in different modalities (mostly text, images, and videos). They also include a restricted data model named Content Model (16).

b) A Multimedia Database Interface (MDI) for accessing this information transparently.

c) A Hyperstructure Adapter (HSA) that generates user-adapted hypermedia document descriptions.

d) The Information Resource Control Structure (IRCS), a repository of mostly generic web pages that include processing rules and links to database entries. IRCS pages are encoded in an extension to HTML (17).

e) The User Model Server (UMS) that hosts models of several users.

f) A User Interface (UI) that presents hypermedia information in a user-tailored way.

\section{Fig. 1 AVANTI architecture}

Fig. 1 gives an example of the basic interaction between these components (the numbers refer to those in the figure):

(1) The user requests a hypermedia page at the user interface. The UI forwards this request to the Hyperstructure Adapter.

(2) The HSA fetches the requested hypermedia page from the IRCS. In most cases, this will be a generic page. Apart from static elements, an IRCS page may contain optional and alternative hypermedia objects ${ }^{3}$, and also groups of hypermedia objects with an associated layout like a page header, toolbar, etc. The processing of these optional and alternative elements is controlled by Adaptation Rules, which can take information from other system components into account, namely assumptions about user characteristics (e.g., knowledge, interests, preferences) from the User Model Server, and content-related information about multimedia objects from the Content Model via the MDI. Information about the current user's session (e.g., previously requested IRCS pages, previously provided input) is also

\footnotetext{
${ }^{3}$ An example for an optional element is supplementary information on wheelchair accessibility. Examples for alternative elements are general vs. more detailed descriptions, and a picture of a painting vs. its textual description.
} 
available from the usage model. A second group of rules that may be contained in an IRCS page are User Model Construction Rules. They control the formation of primary assumptions about the user (i.e., assumptions which are directly derived from the user's interaction with the hypermedia page). Primary assumptions are directly reported to the UMS by the HSA. The HSA interprets the requested hypermedia page and the Adaptation Rules, generates an adapted page (which is compliant to standard HTML) and hands it over to the User Interface for presentation.

(3) The UI interprets the hypermedia page, retrieves multimedia objects from the AVANTI databases transparently via the MDI, and finally presents the requested hypermedia page to the user ${ }^{4}$.

The communication between all active components is carried out via the HTTP protocol. On top of it, a restricted and slightly enhanced version of KQML (Knowledge Query and Manipulation Language $(18,19))$, a high-level communication language and protocol which is independent of the hardware and software platform, is used for communication with the UMS.

The main advantages of this architecture are ${ }^{5}$ :

- Already existing software in the area of the WWW (e.g., communication libraries, browsers, servers, web development environments, and database gateways) could be used for the development of AVANTI components.

- Most WWW browsers available today can access the AVANTI system and take advantage of the individualization features at the content level.

- All components of AVANTI can be distributed according to organizational and technical requirements. These mostly technically motivated decisions do not affect the user's mobility since the user model and the corresponding user model server are always located on the AVANTI system when the user accesses the system.

The HSA and the UMS are principal components of the AVANTI architecture. We did not have to start their development from scratch, but were able to employ already available software as a basis, including WebObjects from Apple Corp. (21) for the HSA, and BGP-MS $(22,23)$ for the UMS.

\section{User and Usage Model}

In order to provide user-oriented adaptivity, a user model is maintained by the AVANTI system which contains assumptions about relevant characteristics of an individual user, including his/her

\footnotetext{
${ }^{4}$ As pointed out before, the UI is able to perform additional adaptations (e.g., use alternative I/O devices, visual and non-visual interface objects and associated interaction techniques) which are not further discussed here.

${ }^{5} \mathrm{An}$ architecture similar in spirit is that in (20), probably for similar reasons.
} 
- interests and preferences, such as interest in accessibility information about buildings (e.g., availability of ramps and elevators), detailed information about the history of sacral buildings, or preferences for certain information modalities (e.g., graphics, video);

- domain knowledge about prominent reference points, such as squares, churches, bridges and the infrastructure in the metropolitan area of interest;

- competence in handling computers, such as the ability to manipulate interface elements within a WIMP (Windows, Icons, Menus, Pointer) interface to achieve a desired goal; and

- competence in handling the AVANTI system, such as the user's knowledge about the orientation and navigation aids and the adaptation mechanisms in AVANTI.

Different methods for acquiring assumptions about the user have been discussed in the literature (24). In AVANTI, assumptions are acquired based on the following sources of information:

- An initial interview allows for the acquisition of primary assumptions (25) about the user and is therefore a valuable source of information for initially assigning the user to certain user subgroups (see the "stereotypes" below).

- Based on primary assumptions about the user and additional information about the application domain, the system can draw inferences in order to acquire further assumptions about the user. For instance, if the user requests more than once detailed information on the history of some churches, he/she can be assumed to be interested in churches, and this detailed information will henceforth be automatically provided.

- Stereotypes (26) contain assumptions about interesting characteristics of user subgroups (e.g., the presumable knowledge of domain experts or the presumable interests of wheelchair-bound users). If certain preconditions are met, a stereotype can be activated for a specific user, which means that the assumptions contained in the stereotype become assigned to the user.

In the following, we briefly present those representational user modeling services offered by BGP-MS that are used in the AVANTI user model server. After that, we describe the user model architecture that is used in the AVANTI information system for the city of Siena, Italy.

BGP-MS's representation component offers two layers for modeling characteristics of individual users and user groups. An inner layer represents the contents of assumptions in the conceptual knowledge representation language SB-ONE (27), which fits loosely into the KLONE paradigm (28). In the outer layer, partitions collect the different types of assumptions about the user or user groups (23). They can be ordered in an inheritance hierarchy, where subordinate partitions inherit the contents from superordinate ones.

\section{Fig. 2 User model in the Siena information system}

Fig. 2 shows the partition hierarchy that is currently used in the AVANTI system for the city of Siena. The model of an individual user consists of the following partitions: 
- SBUI (System Believes User Interests), which contains assumptions about the user's interests,

- $\mathrm{SB} \neg \mathrm{UI}$, which contains assumptions about what the user is not interested in,

- SBUB (System Believes User Beliefs), which contains assumptions about the user's knowledge, and

- $\mathrm{SB} \neg \mathrm{UB}$, which contains assumptions about what the user does not know.

All other partitions belong to the stereotypical part of the user model. The top partitions represent the interest and knowledge stereotypes of "Any User". On the left side of the stereotype hierarchy are partitions for the interests of dystrophic users, wheelchair-bound users and blind users originating from their respective handicaps (e.g., interest in information about the availability of ramps for wheelchair-bound users). The partitions on the right contain stereotypical assumptions for the knowledge of novices and experts about computers, the AVANTI system and the city of Siena. The dashed lines in Fig. 2 exemplify dynamic inheritance relationships in an individual user model between the interest-related stereotypes and SBUI, and the knowledge-related stereotypes and SBUB. Inheritance links are activated or retracted by the stereotype manager of the user model server on the basis of a set of activation/retraction rules that are periodically executed. An example of a simple activation rule used in the AVANTI user model server is:

If the user checks the item "Blind or low vision" in the initial questionnaire, then activate the interest stereotype "Blind User". 6

After the activation of the stereotype "Blind User", all blindness-related interests are inherited by the partition SBUI. Inheritance does not take place if a specific assumption is already contained in SBUI or if its negation is contained in SB $\neg$ UI. For the time being, interests can only be inherited by SBUI since only positive interests are contained in the disability-related stereotypes. In a similar manner, SBUB (but not SB $\neg \mathrm{UB}$ ) can inherit from one or more of the partitions on the right side of the stereotype hierarchy.

In order to be able to adapt to the usage environment, a usage model is maintained by the AVANTI system which contains information about relevant aspects of the user's system usage such as:

- user interface characteristics, to provide alternative task structures according to specific physical and/or sensorial abilities of the current user (29);

- network characteristics, like response time and the available bandwidth.

Apart from information that is available a priori, like the physical environment of a specific terminal or the functionality of a specific type of user interface, most information in the usage model is extracted at run-time from hypermedia page requests via the HTTP protocol (30). Examples include the HTTP header fields "User-Agent", which contains the type of user interface initiating the request, and "Content-Length", which contains the length of the HTML

\footnotetext{
${ }^{6}$ Another (more realistic) option that we tested is to communicate this interest to the AVANTI system via a contactless smartcard that can be read from a distance. Contactless smartcards also provide a satisfactory solution for initially adapting a visual interface and associated hardware to a blind user.
} 
page sent to the UI. An additional source of information for estimating the overall response time from a user's point of view are network probes (31), which are injected as dummy images at the beginning and the end of each hypermedia page sent by the HSA. Based on the estimated response time stored in the usage model, technically motivated adaptations can be applied by the HSA. For instance, if users only have low-bandwidth access to AVANTI then high-bandwidth information like high-resolution pictures and videos is replaced by less demanding, but nevertheless appropriate, equivalents.

\section{The BGP-MS User Model Server}

In AVANTI, user models reside entirely in the User Model Server (UMS). The principal reasons for this include the following:

- The centralized management of user-related information offers the UI and the HSA location-independent access to the most recent user-related information.

- Synergetic effects with respect to user-related information can be exploited (e.g., the UI can take advantage of assumptions acquired by the HSA and vice versa).

- The HSA and the UI can become totally relieved of user modeling tasks and can take advantage of sophisticated run-time services of the UMS like

- the incremental construction of user models based on primary assumptions and dialog acts (25),

- inferences (so-called secondary assumptions) based on primary assumptions,

- the inclusion or exclusion of sets of typical assumptions (stereotypes) about user groups on the basis of activation and deactivation rules, and

- consistency maintenance.

BGP-MS was chosen as a basis for the UMS for several reasons:

- The scalable functionality puts three representation paradigms at the disposal of the user model developer. Only terminological logic has been employed in AVANTI so far. If needed, first order predicate logic or modal logic could be added to enhance the representational and inferential capabilities of the user model.

- The flexible management of stereotypes is supported. This includes a wide range of activation and deactivation rules.

- Different kinds of user information (e.g., interests, knowledge, abilities) can be stored in different representational structures, and efficiently retrieved and employed for inferences.

Nevertheless, BGP-MS has been substantially enhanced in order to establish a network-wide user modeling service. A prototype of the UMS has been made available which offers the following additional features in comparison to (22):

- Access as a network-wide service through a restricted and slightly enhanced version of KQML (27). The chosen implementation KAPI (KQML Application Programmer's Interface (32)) has been substantially revised. Special emphasis has been paid to the name 
service contained therein, which, for example, allows a UI to dynamically locate and access the UMS that hosts the model of a particular user.

- Support for hosting the models of several users at a time.

- Simultaneous support for modal operators representing different assumption types within a user model (e.g., "user interests", "user believes", "user can").

- Availability on Allegro Common LISP for Windows/PC (33). The most recent version of BGP-MS has been ported from CMU Common LISP (34) on SUN workstations to Allegro Common LISP for Windows NT/95/98.

- A framework for a privacy and security architecture is currently under development (35).

Moreover, a domain-specific user modeling system has been implemented for the field trials in AVANTI, which comprises models of individual users and user groups as well as stereotype activation and deactivation rules and inference rules (see Section 5).

\section{Adaptations in the Hyperstructure Adapter}

\subsection{Examples of user-tailored page adaptations}

In order to adapt IRCS pages according to the assumptions stored in the UMS, page descriptions contain information options as well as information alternatives. Depending on the user and the usage model, the system presents optional information if it seems to be beneficial to the user, and chooses the most appropriate among the information alternatives. The decision and selection process is controlled by adaptation rules which are a part of the hypermedia pages. Most of the adaptations that are automatically performed by the system are still under the control of the user (system-initiated user-controlled adaptation in terms of (36)). This enables the user to modify the selection of the provided information if the system's choice should not be appropriate.

Input of the user (which includes his/her feedback to adaptations initiated by the system) as well as navigation behavior is exploited to update the user model by user model construction rules. These rules are also part of the hypermedia pages.

Fig. 3 AVANTI browser: expert user - adaptable table

Fig. 4 AVANTI browser: expert user - table modified by the user

Fig. 5 Netscape browser: novice user - general information about the Duomo

The AVANTI information system for the city of Siena provides hypermedia information for getting around in the city, including special information for people with disabilities. Figs. 3, 4, and 5 show example pages of this system. In the following, we will use them for describing 
the adaptation of IRCS pages in more detail. The pages in Figs. 3 and 4 inform about the services, facilities and amenities that are available in the accommodation "jolly hotel excelsior". The pages can be separated into three columns. The left column with the brickpattern background contains mostly the main menu. The middle column contains the "real" information (e.g., the services offered by a hotel), and the right column includes menu buttons that point to other relevant information.

In their present form, both pages are tailored to a user who is experienced in using computers and the AVANTI system and needs special information for wheelchair-bound and dystrophic people (these needs would come from an initial questionnaire or a contactless smartcard). For instance, the optional navigation path displayed in the upper left corner is only available to experienced web users since its usage requires at least a coarse mental model of the hypermedia space at hand. The navigation aids offered to web novices are restricted to the hierarchical Siena menu on the left hand-side (see Fig. 5) since such users may be confused by alternative navigation elements on a hypermedia page. The hand icon pointing to "restaurants" and the link "nearest restaurants" in the lower right corner are pointing to information that is presumably interesting for the current user. Such an assumption is acquired by the system through inference mechanisms based on the navigation history. For instance, if the user requests more than once detailed information on the cooking style of some restaurant, she/he can be assumed to be interested in restaurants.

Other optional elements are the so-called role-taking buttons in the toolbar of the AVANTI browser. The upper right corner of Figs. 3 and 4 contains the role-taking buttons "Deambul" for deambulation (walking around), "Wheel" for wheelchair, and "Blind" (the buttons have been circled in Fig. 3) ${ }^{7}$. The availability of these buttons is also contingent on the AVANTI expertise of the user. If one or more of these buttons are pressed (e.g., "Deambul" and "Wheel" in Figs. 2 and 3), the user automatically obtains additional information for the selected user groups (e.g., additional information for dystrophic people with difficulties walking around and wheelchair-bound persons, respectively). These buttons were designed for people who look for information on behalf of disabled persons. For instance, travel agency clerks can "take the role" of a wheelchair-bound customer while working with the AVANTI system. From a technical point of view, the role-taking buttons can be used to activate or deactivate certain stereotypes (i.e., "Dystrophic User", "Wheelchair User", "Blind User"). When a role-taking button is switched on or off, the HSA reports the respective key concept or its negation to the UMS which inserts it in the partition SBUI or SB $\neg$ UI. Based on this, stereotype management rules activate or deactivate the corresponding stereotypes.

In AVANTI, tables presented to the user contain those attributes only which the system deems to be relevant for him/her according to the current user model. The user is able to modify the system-initiated attribute selection by requesting an adaptable version of the table that includes all available attributes. Fig. 3 shows such an adaptable version where the user has

\footnotetext{
${ }^{7}$ These buttons, as well as the "Exit" and "Help" buttons in Figs. 3 and 4, are part of the page descriptions coming from the HSA. They are included in the AVANTI browser while its HTML parser is processing the page. Since the HSA behaves like a "normal" web server, it is also possible to access the HSA with any commercial browser. The extra buttons will then remain in the HTML page (like "Exit" and "Help" in Fig. 5).
} 
deselected "Baby-sitting service" and some other attributes and subsequently confirms his/her deselection. Based on this fact, the system does not display these attributes anymore in Fig. 4.

Additionally, the HSA will make assumptions concerning the user's interests and will report them to the UMS (e.g., that the user is not interested in information related to "Baby-sitting service"). The UMS then updates the individual user model (e.g., inserts the related concept into $\mathrm{SB} \neg \mathrm{UI}$ ) and will possibly activate or deactivate some of its stereotypes. When conflicts between assumptions arise, those coming from stereotypes have a lower priority than those directly obtained from the interaction with the user. If there are too many contradictions between the individual user model and an activated stereotype (e.g., if the user is only interested in less than $10 \%$ of the stereotype concepts), the stereotype becomes a candidate for deactivation: in Fig. 4, the system encourages the user to reconsider his/her interest in wheelchair-bound people and dystrophic people, due to his/her attribute selection in Fig. 3. If the user confirms not to be interested anymore, the corresponding interest stereotypes will become deactivated. A confirmation by the user is necessary since the retraction of these stereotypes will again have considerable impact on the content of hypermedia pages, their links to other pages, and the user interface: if the user confirms the system's assumption in Fig. 4, attributes like "Link with shared parts" will not be displayed anymore, the button "Interior access" which leads to more specific information for disabled people will get removed, and the activated role-taking buttons will be switched off by the system.

Based on the user's answer in the initial interview that he/she has never been in Siena before, the system automatically presents rather general information about the Duomo (see Fig. 5). The user can request a more detailed alternative description by pressing the "+" button. User requests for more detailed or more general descriptions also refine the user model (e.g., if the user requests more than once detailed information on the history of some churches, she/he can be assumed to be interested in churches, and the corresponding concept will be inserted in the individual user model partition SBUI).

\subsection{The authoring environment for user-adaptive IRCS pages}

We now look closer into the internal structure of IRCS pages and how they are written by authors, using the optional link "Interior access" as an example. IRCS pages are based on the server-based development environment "WebObjects" from Apple Computer Inc. (21). Fig. 6 shows the WebObjects authoring environment including a small part of the IRCS page which is visualized in Fig. 4.

The background window in Fig. 6 shows a graphical representation of the IRCS elements that correspond to the three columns of the final page layout in Fig. 4: the left column with the brick-pattern background shows parts of the main menu, the second column presents part of the table, and the third column with the circled optional element contains the link to information on "Interior access" that we will study further below.

The foreground window of Fig. 6 shows the associated functionality with its content presented in different parts of the window. The first column of the upper part presents the different categories of the application resources. Since the category "Web Components" has been 
selected, all Web Components are listed in the second column. An AVANTI IRCS page is represented by such a WebObjects component ("hotel.wo" in our example) and contains the following three files:

- An HTML template file ("hotel.html" in our example) which specifies the content and the overall layout of the IRCS page in HTML. Tags define the format for both static and dynamic page contents. Dynamic page contents are marked up with the special "WEBOBJECT" tag which refers either to functionality or to components defined outside of the current IRCS page.

- A declaration file ("hotel.wod" in our example) which defines a mapping between the WEBOBJECT tags in the HTML template and their associated functionality.

- A script file ("hotel.wos" and "session.wos" in our example) which implements application behavior specific to the component. Script files manage dynamic page generation and respond to user requests. They are written in WebScript, the WebObjects scripting language. WebScript is a subset of the object-oriented language Objective-C.

In most cases, script files belong to one component. However, session-wide and applicationwide resources are stored in separate files called "session.wos" and "application.wos", respectively.

Let us now concentrate on the correspondence between the fragments in the development environment and their hypermedia counterparts after being processed by the HSA. In Fig. 4, the link "Interior access" (in the lower right corner of the screen) is only available if the user is assumed to be interested in information for disabled people. The internal structure of this optional element with its assigned adaptation rule is presented in Fig. 6: the circled part of the background window shows the element in graphical form, while the same information is given in more detail in the component files displayed in the editor of the foreground window.

The outer syntactical element in the graphical pane (indicated by the opening and closing question mark) is a conditional called "IfInterestInInformationForDisabled". Within this conditional, we see a hyperlink which refers to another page (indicated by the opening and closing chain symbol) and an image with associated text (indicated by the opening and closing star and the opening and closing symbols around the "imageString", respectively).

In the template file hotel.html, we see a WebObject HTML tag corresponding to IfInterestInInformationForDisabled. The mapping of this tag to a conditional WebObjects element and its associated session method IfInterestInInformationForDisabled can be found in the declaration file hotel.wod. Finally, in session.wos we find the implementation of IfInterestInInformationForDisabled, which queries a user model object for the user's interest in information for disabled people (i.e., whether the current user is interested in information for dystrophic people, blind, or wheelchair-bound persons). More specifically, the method checks whether one of the key concepts "dystrophy_information", "wheelchair_information", or "blind_information" is contained in the partition SBUI of the individual user model. Access to the user model is established via a user model object (i.e. "umObjectUser"), which encapsulates the complexity of KQML communication, networking, and protocol issues. 
Therefore, an appropriate level of abstraction is provided to the hypermedia author (in fact, the user model is hosted by the remote user model server).

Since most of the images depend on the language selected by the user during runtime, the HTML image tag and the corresponding alternative text (i.e., "ALT TEXT") has to be generated dynamically. The component "ImageTool" which manages images according to the selected language can be employed for this purpose on every IRCS page. ImageTool is an example of a reusable component, which can be regarded as a building block for the dynamic parts of an IRCS page. Reusable components can be nested; in this case, they serve as building blocks for other reusable components. In addition to reusable components developed in AVANTI, reusable functionality is also provided through methods and objects that can be defined as session-wide or application-wide (e.g., the session-wide method IfInterestInInformationForDisabled mentioned earlier).

The IRCS pages developed in AVANTI take advantage of AVANTI-specific classes and components. The IRCS author is provided with an appropriate level of abstraction, and lowlevel coding can be omitted ${ }^{8}$. Since all this enhancements are encapsulated within the HSA and the underlying WebObjects development environment, the compatibility with existing and upcoming standards (e.g., HTML and XML, respectively) is preserved. For the other AVANTI subsystems (e.g., the UI), the HSA behaves like a "normal" web server.

\subsection{Adaptation on the User Interface Level}

Adaptation in AVANTI does not only take place on the level of hypermedia pages, but also on the user interface level. This is particularly true for the selection of certain input and output devices and media, as well as the behavior of a number of user interface elements. These types of adaptation are performed by the AVANTI User Interface and are described in detail in (14). We want to give a brief example of how the modality and the navigation elements become adapted for blind people in AVANTI on the page description level.

Fig. 7a presents a map of the city of Siena which includes all interesting places like museums, churches, restaurants, and hotels. The Siena map can be accessed via the "city map" button in the upper left corner on every page (see Figs. 3, 4, and 5). .9 The "same" page for blind people is presented in Fig. 7b. While the visual map of Siena gives an overview of the different locations and allows users to figure out how to go there, the "map" for the visually impaired

\footnotetext{
${ }^{8}$ The adaptive hypermedia system AHA (37) also employs generic web pages with alternative and optional elements, which become pre-processed by the AHA engine before being transferred to the web browser. However, this system lacks the graphical editing environment of AVANTI, the object-oriented development environment for specifying adaptive behavior, and the opportunity to turn arbitrary parts of an IRCS page into a reusable component. In AHA, authors specify layout and dynamic behavior in (extended) HTML. Another important difference is scalability. As a commercial product, WebObjects allows for transparently dispatching incoming page requests to several AVANTI applications, which may be located on several networked computers. Thereby, an increasing number of users does not necessarily increase the overall response time of the system.

${ }^{9}$ The label "city map" is only presented for web novices (see Fig. 5) and is omitted for experienced web users (see Figs. 3 and 4).
} 
contains textual descriptions of all important locations relative to the Piazza del Campo, the central square in Siena. ${ }^{10}$ To provide a better overview for the blind, pages must be kept short and separated into categories (i.e. museums, churches, restaurants, and hotels). ${ }^{11}$ An additional element available at the top of every page for blind people is the "Short Summary". Since the blind cannot skim a page like normal-sighted people, this short summary must contain sufficient information to allow them to decide whether the page seems to be relevant .

\section{Evaluation}

The AVANTI system has been extensively evaluated at three different sites in three different scenarios. In this section we will summarize the evaluation results as reported in $(38,39)$. The common question shared by all experiments is whether the developed system is beneficial for users, and specifically whether it is technically feasible and useful for end users to introduce adaptability and adaptivity in the information contents and user interfaces of hypermedia information systems.

The trials were carried out a three different sites:

- The experiments in Siena were performed with 60 citizens and tourists, including people with motor disabilities. Test sites were the University of Siena School of Engineering, a public information point at a multimedia center, a motor-impaired citizen's home, and a travel agency. A laboratory case study on 4 blind users has also been performed at CNR. Subjects received tasks like planning sightseeing trips to Siena, planning to attend a music festival there, planning errands in town as a citizen, planning a field study to see the cathedral floor (which is only uncovered twice a year), and planning a group tour for people with mobility impairments by travel agency clerks.

- The experiments in Kuusamo, Finland were carried out with 11 random visitors of a tourist center, and in a second stage with 40 tourists, business travelers and travel agency clerks. All subjects were able-bodied. The tasks consisted in booking a vacation cottage in this tourist region, either for oneself or for a client.

- The trials in Rome, Italy were conducted with 20 visually impaired users and 40 users without visual impairments. They comprised tourists, pilgrims, and citizens of Rome. Tasks included looking for information on the Fontana dei Trevi and St. Peter's Basilica, and searching for the location and for directions to the municipal lost-property office,

In all three cases, the systems' adaptation features outnumbered the adaptivity features.

Users were subject to usability test procedures to determine the system's learnability, efficiency of use and memorizability, users' error-proneness, satisfaction and overall attitude, and the specific contribution of adaptability and adaptivity. Data were collected through observation, interviews, questionnaires and the analysis of $\log$ files. Selected findings that

\footnotetext{
${ }^{10}$ Such alternative descriptions for graphical elements, e.g., in the HTML "ALT TEXT" tag, are compulsory when defining AVANTI IRCS pages.

${ }^{11}$ Another approach is realized in the WAB system which creates intra-page links at the top of each page to facilitate the exploration of long pages with several paragraphs (7).
} 
refer to adaptation features or individual differences between users include the following ones (most are qualitative):

- Repeat AVANTI users better understand and use the adaptation features.

- While adaptability features have been recognized and understood by most users, they have been more frequently used by AVANTI experts (namely adaptive tables), and computer and web experts (namely the path navigation tool).

- Motion-impaired users were generally more satisfied with the information provided by the system and had a better attitude towards AVANTI than able-bodied users. ${ }^{12}$ They found the system properly designed for them and the information on accessibility very clear and useful for mobility. They also appreciated very much the level of detail reached by the system, and many of them praised the system enthusiastically.

- In contrast, able-bodied user did not easily find information for their purposes (and often this kind of information was not present in the system). It also seems that their concept of mobility differs from that of impaired users in several ways, and was not properly reflected in the system.

- Shortcuts to frequently visited pages would automatically pop up in the index frame of the Kuusamo web pages. Most users who noticed them did not understand their meaning. When told, all felt that it was a useful feature of the system.

- Travel agency clerks appreciated the possibility to create their own shortcuts.

- Additional accessibility buttons and role-taking buttons were generally well recognized, correctly understood, and frequently used.

The results allow the conclusion that AVANTI's adaptation features were generally well understood, used, and appreciated (some of them were not self-explanatory though). The benefit for information systems probably lies more in user satisfaction than in efficiency gain or error reduction (which would be difficult to measure anyway in representative in vivo experiments). In the AVANTI successor project HIPS, we expect to gain further insights into the usefulness of personalization in touristic domains from the evaluation of the HIPS museum guide $(40,41)$. This portable system adapts information based on the current location of the visitor and his/her presumable interests as inferred from his/her navigation path through the museum.

\section{Summary}

An adaptable and adaptive hypermedia information system has been presented that caters not only to able-bodied, but also to several kinds of disabled users. "Access for All" to hypermedia systems seems to be very important since this medium is likely to play the role of a gateway to electronic information and services in the next few years, both in professional and domestic environments. The results of AVANTI show that extending the current adaptability and adaptivity mechanisms in such a way that users' physical, sensory, and

\footnotetext{
${ }^{12}$ The difference was very significant when comparing users' first session with the system (Fisher's PLSD, $\mathrm{p}<$ 0.001 ).
} 
cognitive abilities as well as their technical environments are taken into account is technically feasible and well appreciated by users. This approach seems to be more promising than providing post factum access for handicapped user groups for whom the system was originally not designed.

\section{References}

1. AVANTI Home Page. 1998. Available at http://www.avanti-acts.org/.

2. MCCOY, K., PENNINGTON, C., and SURI, L. English Error Correction: A Syntactic User Model Bases on Principled "Mal-Rule" Scoring. Proceedings of the Fifth International Conference on User Modeling, Kailua-Kona, Hawaii, 1996, 59-66.

3. GORI, M., MAGGINI, M., and MARTINELLI, E. Web-Browser Access Through Voice Input and Page Interest Prediction. Proceedings of the Sixth International Conference on User Modeling. Wien, New York, Springer: 1997, 17-19.

4. TREWIN, S., and PAIN, H. Dynamic Modelling of Keyboard Skills: Supporting Users With Motor Disabilities. Proceedings of the Sixth International Conference on User Modeling. Wien, New York: Springer, 1997, 135-146.

5. SPOONER, R. I. W., and EDWARDS, A. D. N. User Modelling for Error Recovery: A Spelling Checker for Dyslexic Users. Proceedings of the Sixth International Conference on User Modeling. Wien, New York: Springer, 1997, 147-157.

6. BINI, A. User Groups' Needs and Requirements. AVANTI Deliverable AC042-DE001 (Project AVANTI), Brussels, 1996.

7. KENNEL, A., PERROCHON, L., and DARVISHI, A. WAB: World-Wide Web Access for Blind And Visually Impaired Computer Users. New Technologies in the Education of the Visually Handicapped. ACM SIGCAPH Bulletin, June 1996. Available at http://www.inf.ethz.ch/department/IS/ea/blinds/.

8. CONKLIN, J. Hypertext: An Introduction and Survey. IEEE Computer, September 1987, 17-41.

9. NIELSEN, J. The Art of Navigating through Hypertext. Communications of the ACM, vol. 33, no. 3, 1990, 296-310.

10. SCHAUMBURG, H., and ISSING, L. J. Lernen mit Hypermedia: Verloren im Hyperraum? HMD - Theorie und Praxis der Wirtschaftsinformatik. No. 190, 1996, 108121.

11. NIELSEN, J. Usability Engineering. San Diego, CA: Academic Press Inc., 1993.

12. OPPERMANN, R. (Ed.). Adaptive User Support - Ergonomic Design of Manually and Automatically Adaptable Software. Hillsdale, NJ: Lawrence Erlbaum Associates, Inc., Publishers, 1994.

13. SAVIDIS, A., and STEPHANIDIS, C. Developing Dual User Interfaces for Integrating Blind and Sighted Users: the HOMER UIMS. Proceedings of the CHI'95 Conference on Human Factors in Computing Systems, Denver, CO, 1995, 106-113. 
14. STEPHANIDIS, C., PARAMYTHIS, A., SFYRAKIS, M., STERGIOU, A., MAOU, N., LEVENTIS, A., PAPAROULIS, G., and KARAGIANIDIS, C. Adaptable and Adaptive User Interfaces for Disabled Users in the AVANTI Project. In: S. Triglia, A. Mullery, M. Campolargo, H. Vanderstraeten, M. Mampaey, eds.: Intelligence in Services and Networks: Technology for Ubiquitous Telecom Services. Berlin, Heidelberg, New York: Springer, 1998, 153-166.

15. BRUSILOVSKY, P. Methods and Techniques of Adaptive Hypermedia. User Modeling and User-Adapted Interaction 6(2-3), 1996, 87-129.

16. FINK, J., RELLA, L., GRIECO, E., BELLINI, A., BONIFACIO, A., and NILL, A. Modeling of Information in the Multimedia DB. Deliverable AC042-DE017 (project AVANTI), Brussels, 1997.

17. World Wide Web Consortium. HyperText Markup Language. 1997. Available at http://www.w3.org/MarkUp/.

18. FININ, T. W., WEBER, J., WIDERHOLD, G., GENESERETH, M., FRITZSON, R., MCKAY, D., MCGUIRE, J., PELAVIN, R., SHAPIRO, S., and BECK, C. Specification of the KQML Agent-Communication Language. 1993. Available at http://www.cs.umbc.edu/kqml/papers/kqmlspec.ps.

19. KOBSA, A., FINK, J., and POHL, W. A Standard for the Performatives in the Communication between Applications and User Modeling Systems (draft). 1996. Available at http://zeus.gmd.de/ kobsa/rfc.ps

20. GATES, K. F., LAWHEAD, P. B., and WILKINS, D. E. Towards an Adaptive WWW: A Case Study in Customized Hypermedia. In this issue.

21. Apple Computer, Inc. WEBOBJECTS 3.5/ENTERPRISE OBJECTS 2.2 DOCUMENTATION, 1998. Available at http://developer.apple.com/techpubs/webobjects/WebObjects_3.5/WebObjectsTOC.HTML

22. KOBSA, A., and POHL, W. The User Modeling Shell BGP-MS. User Modeling and User-Adapted Interaction 4(2), 1995, 59-106.

23. POHL, W. Logic-Based Representation and Reasoning for User Modeling Shell Systems. User Modeling and User-Adapted Interaction 9, 1999 (to appear).

24. CHIN, D. N. Acquiring User Models. Artificial Intelligence Review 7, 1993, 185-197.

25. POHL, W., KOBSA, A. and KUTTER, O. User Model Acquisition Heuristics Based on Dialogue Acts. Proceedings of COOP'95: International Workshop on the Design of Cooperative Systems, Antibes-Juan-les-Pins, France, 471-486, 1995.

26. RICH, E. User Modeling via Stereotypes. Cognitive Science, 3, 1979, 329-354.

27. KOBSA, A. (1991): Utilizing Knowledge: The Components of the SB-ONE Knowledge Representation Workbench. In: J. Sowa, ed.: Principles of Semantic Networks: Explorations in the Representation of Knowledge. San Mateo, CA: Morgan Kaufmann.

28. BRACHMAN, R. J. and Schmolze, J. G. (1985): An Overview of the KL-ONE Knowledge Representation System. Cognitive Science 9(2), 1985, 171-216. 
29. STEPHANIDIS, C., KARAGIANNIDIS, C., PARAMYTHIS, A., SFYRAKIS, M., STERGIOU, A., SAVIDIS, A., SCHRECK, J., and FINK, J. Report on Rules. Deliverable AC042-DE012 (Project AVANTI), Brussels, 1997.

30. FIELDING, R., GETTYS, J., MOGUL, J., FRYSTYK, H., and BERNERS-LEE, T. Request for Comments: 2068, Category: Standards Track. 1997. Available at http://www.w3.org/Protocols/rfc2068/rfc2068.

31. FULLER, R., and DE GRAAFF J. J. Measuring User Motivation from Server Log Files. Proceedings of the Microsoft conference "Designing for the Web - Empirical Studies", Redmond, WA, 1996. Available at http://www.microsoft.com/usability/webconf.htm.

32. KAPI. 1996. Available at ftp://cdr.stanford.edu/pub/JATLite/

33. Franz Inc. ALLEGRO CL for Windows. Berkeley, CA, 1995.

34. MACLACHLAN, R. A. (ed.) CMU Common Lisp User's Manual. School of Computer Science, Carnegie-Mellon University, Pittsburg, PA, 1992.

35. FINK, J., KOBSA, A., and SCHRECK J. Personalized Hypermedia Information through Adaptive and Adaptable System Features: User Modeling, Privacy and Security Issues. In: A. Mullery, M. Besson, M. Campolargo, R. Gobbi and R. Reed, eds.: Intelligence in Services and Networks: Technology for Cooperative Competition. Berlin Heidelberg: Springer, 1997, 459-467.

36. DIETERICH, H., MALINOWSKI, U., KÜHME, T. and SCHNEIDER-HUFSCHMIDT, M. State of the Art in Adaptive User Interfaces. In: M. Schneider-Hufschmidt, T. Kühme and U. Malinowski, eds.: Adaptive User Interfaces: Principle and Practice. Amsterdam: North Holland, 1993.

37. DE BRA, P., and CALVI, L.: AHA! An Open Adaptive Hypermedia Architecture. In this issue.

38. ANDREADIS, A., GIANNETTI, L., MARCHIGIANI, E., RIZZO, A., SCHIATTI, E., and TIBERIO, M. Experimentation Results in Italy. Deliverable AC042-DE027 (project AVANTI), 1998a.

39. ANDREADIS, A. GIANNETTI, L, MARCHIGIANI, E., RIZZO, A., SCHIATTI, E. TIBERIO, M., PENTILA, M., PERALA, J., EMILIANI, P., BINI, A., NILL, A., SABBIONE, A., SFYRAKIS, M., STARY, CH., and TOTTER, A. Global Evaluation of the Experiments. Deliverable AC042-DE030 (project AVANTI), 1998b.

40. OPPERMANN, R. \& SPECHT, M. Adaptive support for a mobile museum guide. Proceedings of Interactive Applications of Mobile Computing 98, Rostock, Germany. Available at http://www.egd.igd.fhg.de/ imc98/Proceedings/imc98-SessionMA3-2.pdf, 1998.

41. NOT, E., PETRELLI, D., SARINI, M., STOCK, O., STRAPPARAVA, C., and ZANCANARO, M. Hypernavigation in the Physical Space: Adapting Presentations to the User and to the Situational Context. In this issue. 


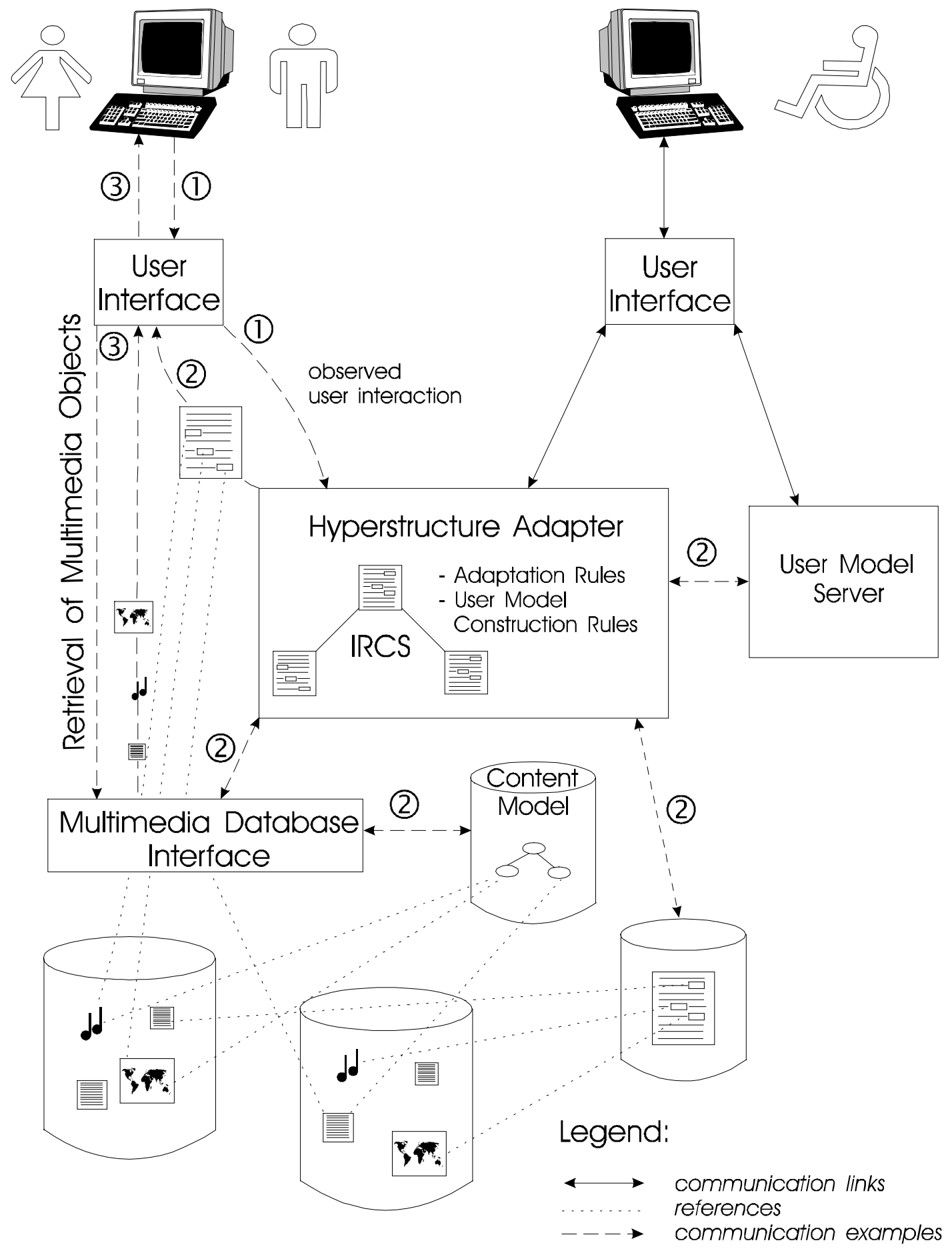

Fig. 1 AVANTI architecture 


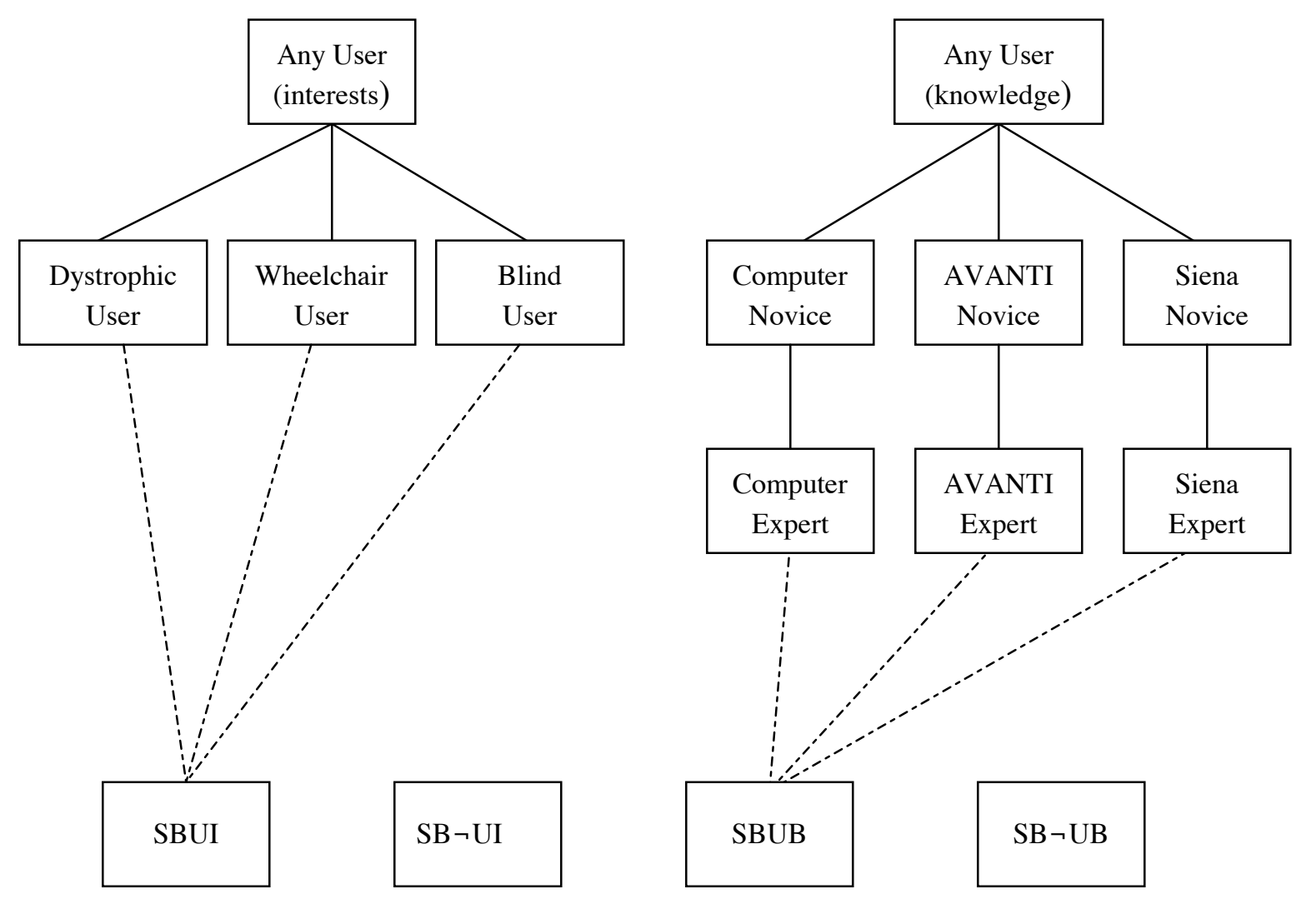

Fig. 2 Partition hierarchy in BGP-MS 


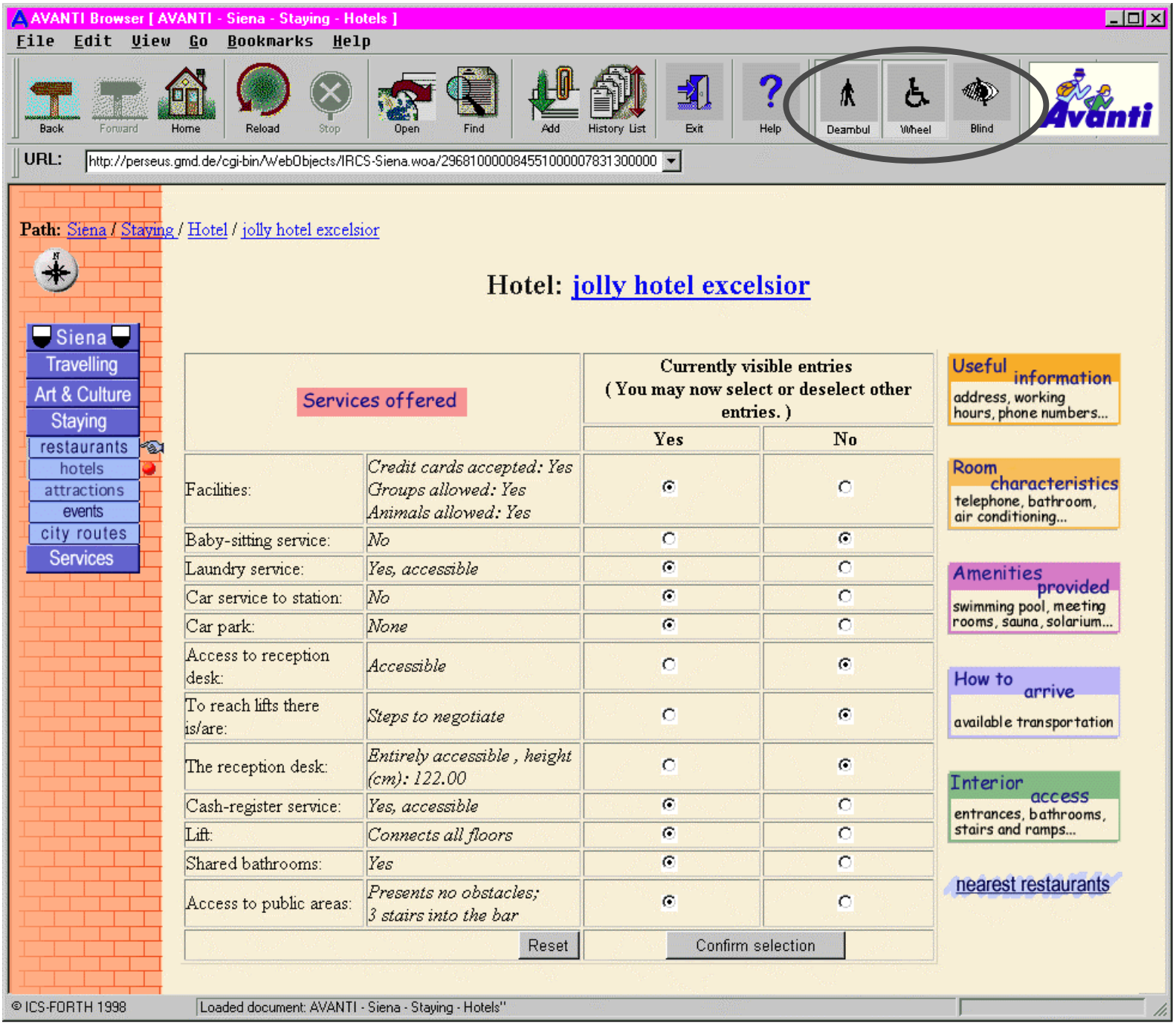

Fig. 3 AVANTI browser: expert user - adaptable table 


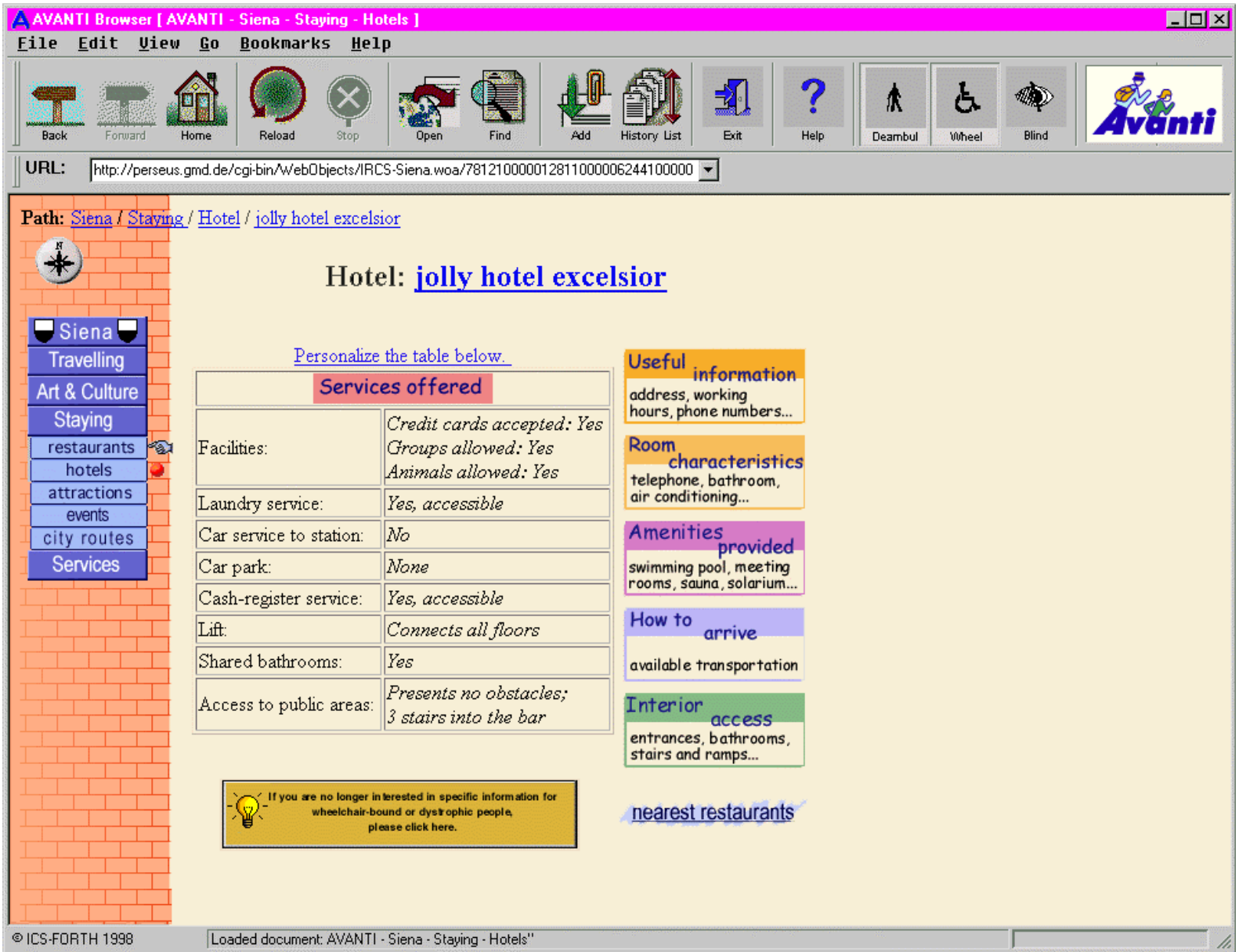

Fig. 4 AVANTI browser: expert user - table modified by the user 


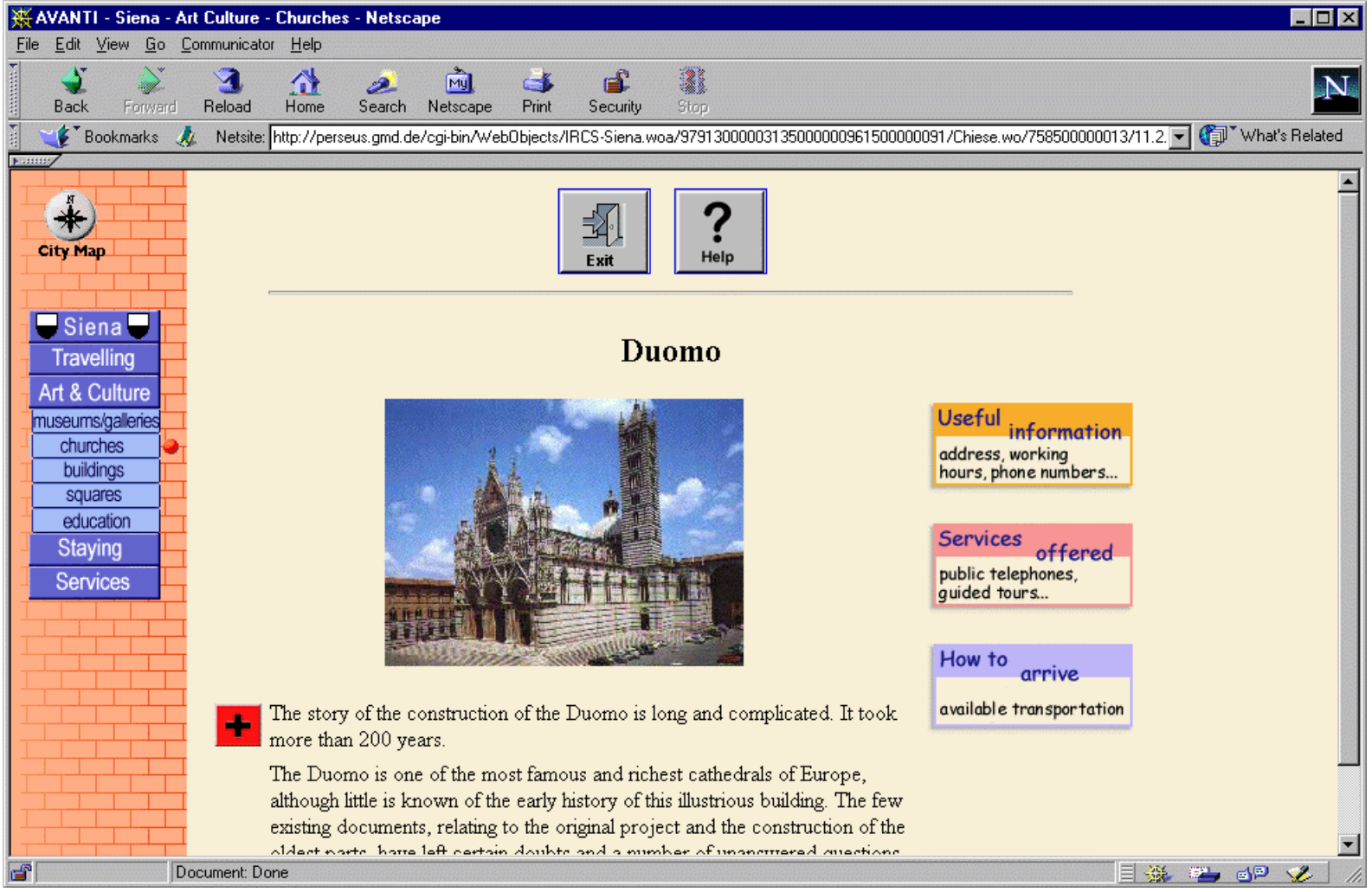

Fig. 5 Netscape browser: novice user - general information about the Duomo 


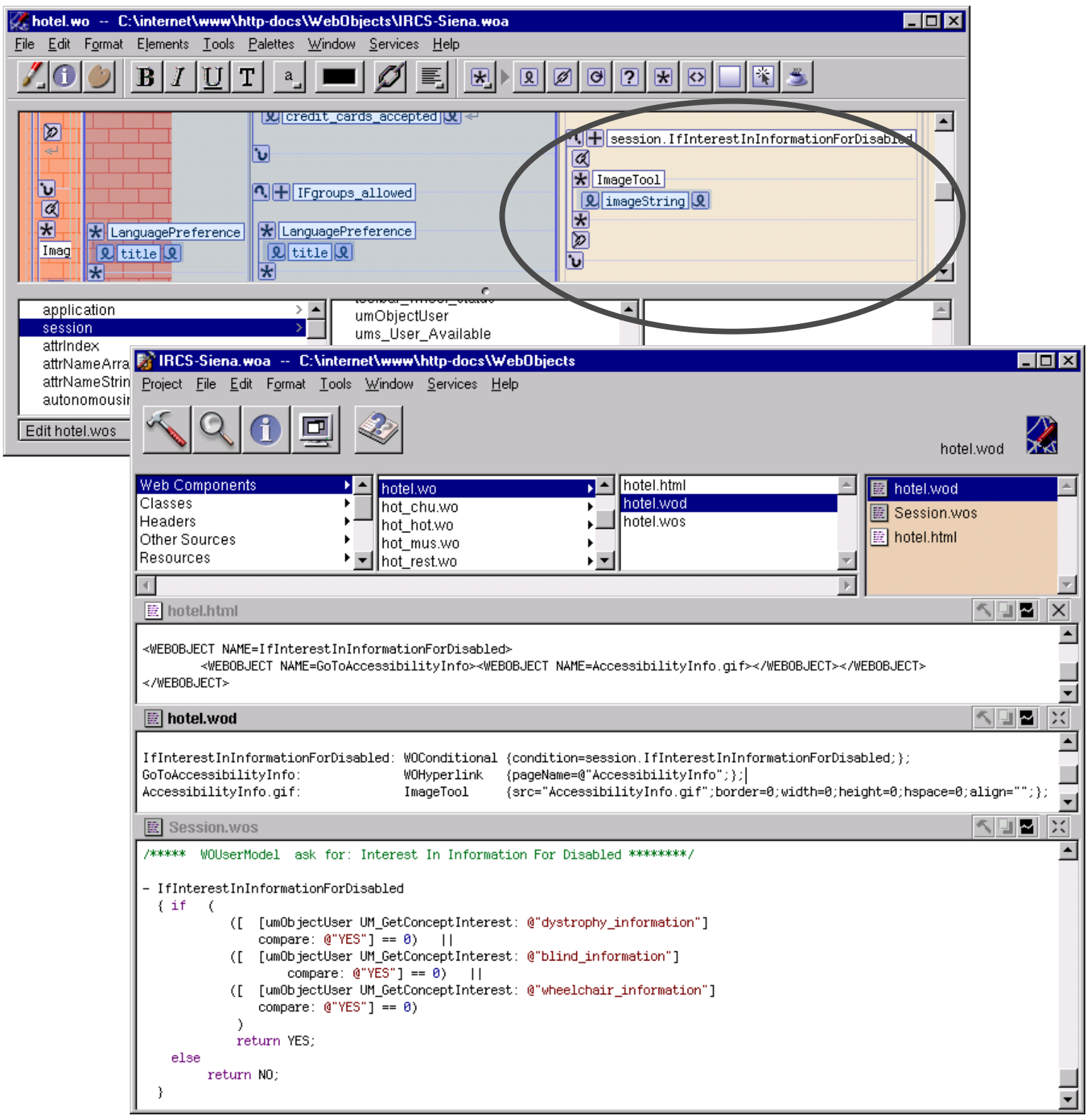

Fig. 6 Developing IRCS pages using the WebObjects development environment 


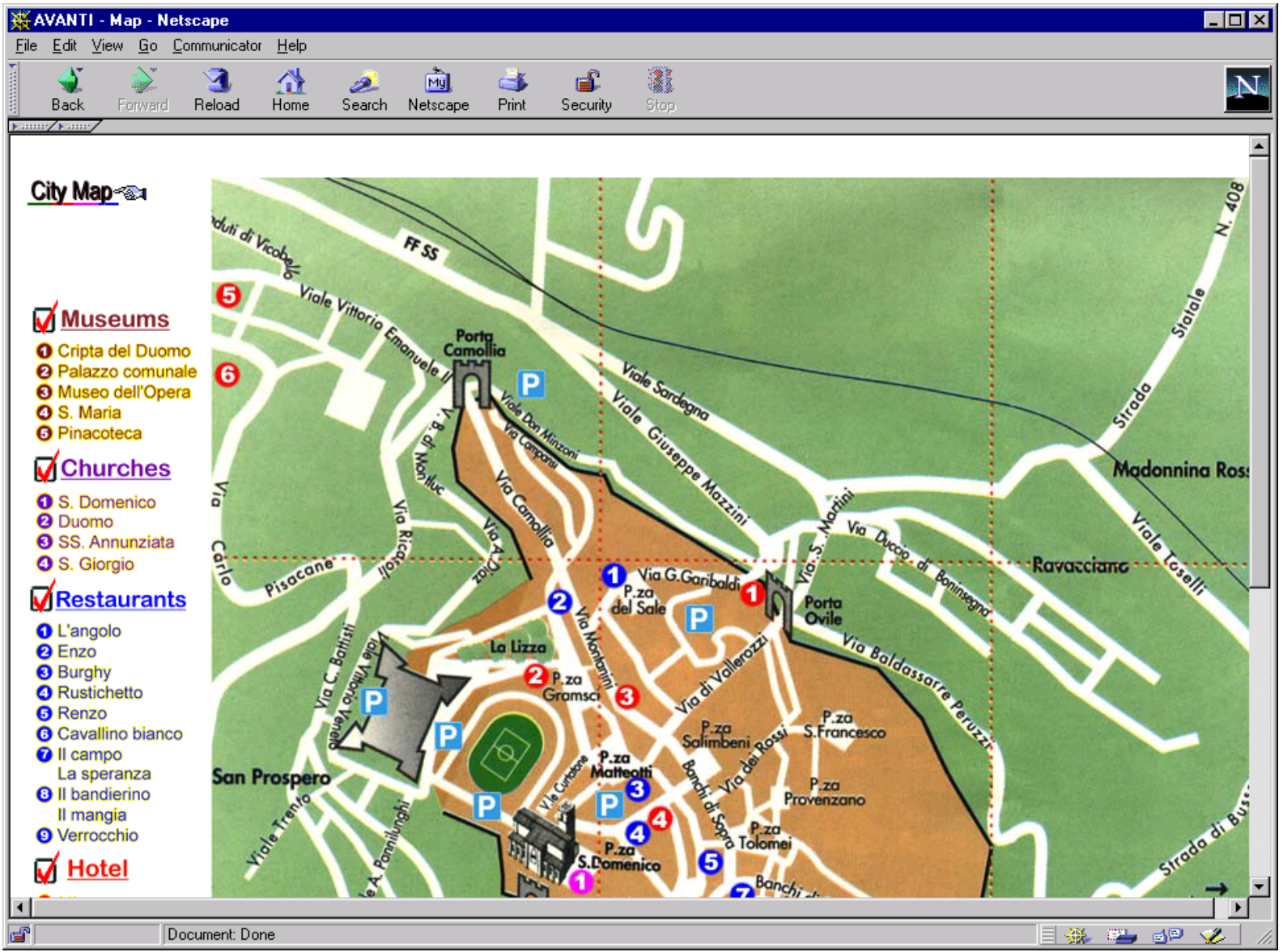

Fig. 7a Siena map in graphical form 
Fi:i:i:

Short Summary: list of the most important restaurants with information on the location, the distance from the piazza del campo, and the accessibility for blind people. If you follow a link to a single restaurant, you will get detailed information on the selected restaurant.

\section{Cily Map}

\section{Museums}

\section{Churches}

\section{VúRestaurants}

(1) L'angolo

2 Enzo

3 Burghy

(4) Rustichetto

6 Renzo

6 Cavallino bianco

(⿻ II campo

location: via garibaldi. 900 meters from piazza del campo to the north. Accessible for blind people location: via di camollia. 1000 meters from piazza del campo to the north. Accessible for blind people. location: piazza matteotti. 700 meters from piazza del campo to the north. Accessible for blind people. location: piazza san giovanni. 500 meters from piazza del campo to the north-west. Accessible for blind people. location: via delle terme. 200 meters from piazza del campo to the north. Accessible for blind people.

La speranza location: via di cita. 200 meters from piazza del campo to the west. Accessible for blind people.

8 II bandierino location plazza del campo. Accessible for blind people.

II mangia location: piazza del campo. Accessible for blind people

9 Verrocchio cation: piazza del campo. Accessible for blind people. location: piazza del campo. Accessible for blind people.

\section{$\underline{\text { Hotel }}$}

Fig. 7b “Siena map” for blind people 